\title{
Gamma-ray emission from the globular clusters Liller 1, M80, NGC 6139, NGC 6541, NGC 6624, and NGC 6752
}

\author{
P. H. T. Tam ${ }^{1}$, A. K. H. Kong ${ }^{1,4}$, C. Y. Hui ${ }^{2}$, K. S. Cheng ${ }^{3}$, C. Li $^{3}$, and T.-N. Lu ${ }^{1}$ \\ 1 Institute of Astronomy and Department of Physics, National Tsing Hua University, \\ Hsinchu, Taiwan \\ 2 Department of Astronomy and Space Science, Chungnam National University, Daejeon, \\ South Korea \\ 3 Department of Physics, University of Hong Kong, Pokfulam Road, Hong Kong \\ ${ }^{4}$ Golden Jade Fellow of Kenda Foundation, Taiwan \\ phtam@phys.nthu.edu.tw
}

\begin{abstract}
Globular clusters (GCs) are emerging as a new class of $\gamma$-ray emitters, thanks to the data obtained from the Fermi Gamma-ray Space Telescope. By now, eight GCs are known to emit $\gamma$-rays at energies $>100 \mathrm{MeV}$. Based on the stellar encounter rate of the GCs, we identify potential $\gamma$-ray emitting GCs out of all known GCs that have not been studied in details before. In this paper, we report the discovery of a number of new $\gamma$-ray GCs: Liller 1, NGC 6624, and NGC 6752, and evidence for $\gamma$-ray emission from M80, NGC 6139, and NGC 6541, in which $\gamma$-rays were found within the $\mathrm{GC}$ tidal radius. With one of the highest metallicity among all GCs in the Milky Way, the $\gamma$-ray luminosity of Liller 1 is found to be the highest of all known $\gamma$-ray GCs. In addition, we confirm a previous report of significant $\gamma$-ray emitting region next to NGC 6441. We briefly discuss the observed offset of $\gamma$-rays from some GC cores. The increasing number of known $\gamma$-ray GCs at distances out to $\sim 10 \mathrm{kpc}$ is important for us to understand the $\gamma$-ray emitting mechanism and provides an alternative probe to the underlying millisecond pulsar populations of the GCs.
\end{abstract}

Subject headings: Gamma rays: stars - Globular clusters: individual (Liller 1, M80, NGC 6139, NGC 6441, NGC 6541, NGC 6624, and NGC 6752) - Pulsars: general 


\section{Introduction}

Globular clusters (GCs) are very efficient in forming close binary systems and their decedents such as low-mass X-ray binaries (LMXBs), cataclysmic variables, and millisecond pulsars (MSPs). It is commonly believed that the high stellar encounter rate of GCs facilitates the formation of these binary systems through dynamical interactions (e.g., Verbunt \& Hut 1987). Pooley et al. (2003) and Pooley \& Hut (2006) have shown by X-ray observations that the number of close binary systems in a GC scales with the stellar encounter rate $\left(\Gamma_{\mathrm{c}}\right)$ of the GC. Recently, Hui et al. (2010) found that a cluster with higher $\Gamma_{c}$ and metallicity hosts more MSPs.

Radio and X-ray observations have revealed about 140 MSPs in 26 globular clusters 1 (Manchester et al. 2005). However, the presence of much stronger X-ray emitters can contaminate the X-ray observations of MSPs. Abdo et al. (2009b) reported a number of MSPs in the Galactic field using data obtained using the Fermi Gamma-ray telescope. Because MSPs are the only known steady $\gamma$-ray sources in GCs, $\gamma$-ray observations serve as an alternative channel in studying the underlying MSP populations in GCs.

The Large Area Telescope (LAT), onboard the Fermi Gamma-ray Space Telescope, is a pair-production telescope designed to detect $\gamma$-rays with energies between $\sim 20 \mathrm{MeV}$ and $>300 \mathrm{GeV}$. It operates in a survey mode in which it scans the whole sky every 3 hours. The $68 \%$ containment radius of individual photons is 0.6 at $1 \mathrm{GeV}$, the typical energy of the photons collected from the GCs. The point-source sensitivity of LAT is $\sim 10^{-8} \mathrm{ph} \mathrm{cm}^{-2} \mathrm{~s}^{-1}$ above $100 \mathrm{MeV}$ in one year of survey-mode observations (Atwood et al. 2009). Such sensitivity has enabled the discovery of $\gamma$-rays from 8 GCs (Abdo et al. 2010b,c), including 47 Tucanae (Abdo et al. 2009a) and Terzan 5 (Kong et al. 2010). From the known $\gamma$-ray luminosity of individual MSPs in the Galactic field (Abdo et al. 2009b), they would not be detected at distances of several kpc. It is therefore believed that the $\gamma$-rays from GCs do not come from individual MSP, but from a population of MSPs.

The radiation mechanism of $\gamma$-rays remains unclear. In the pulsar magnetosphere model (e.g., Venter \& de Jager 2008), $\gamma$-rays up to a few $\mathrm{GeV}$ is radiated from the MSPs through curvature radiation. On the other hand, inverse Compton (IC) processes resulted from energetic particles up-scattering low-energy photons, such as starlight and infrared light, may give rise to $\gamma$-rays of MeV to TeV energies (Bednarek \& Sitarek 2007; Cheng et al. 2010). It is worth noting that $\gamma$-ray observations at $>100 \mathrm{GeV}$ have not yet resulted in any detections (Anderhub et al. 2009; Aharonian et al. 2009).

\footnotetext{
${ }^{1}$ http://www.naic.edu/ ${ }^{\text {pfreire/GCpsr.html }}$
} 
In this work, we searched for $\gamma$-rays from those GCs with high encounter rate. We report the discovery of $\gamma$-rays from the GCs Liller 1, M80, NGC 6139, NGC 6541, and NGC 6752.

\section{Cluster sample and Fermi Observations}

It has long been suggested that the stellar encounter rate of a GC is a measure of the number of LMXBs, the precedents of MSPs (see, e.g. Verbunt \& Hut 1987; Gendre et al. 2003). Assuming that each MSP emits similar amount of $\gamma$-rays, the expected $\gamma$-ray luminosity of a GC is proportional to the number of MSPs it hosts, and thus to the encounter rate $\Gamma_{c}$, as supported by the findings made by Abdo et al. (2010c). Here we estimate the encounter rate by $\Gamma_{\mathrm{c}} \propto \rho_{0}^{2} r_{\mathrm{c}}^{3} / \sigma$ where $\rho_{0}$ is the central luminosity density, $r_{\mathrm{c}}$ the core radius, and $\sigma$ the velocity dispersion at the cluster center. We adopted the values of $\sigma$ as presented in Gnedin et al. (2002), and those of $\rho_{0}$ and $r_{\mathrm{c}}$ in Harris (1996, 2003 version). Then we ranked the GCs according to the encounter rate divided by the squared distance compiled in Harris (1996, 2003 version), giving a measure of the relative expected $\gamma$-ray flux for each GC. Most of the eight known $\gamma$-ray GCs (47 Tucanae, $\omega$ Centauri, M62, NGC 6388, Terzan 5, NGC 6440, M28, and NGC 6652) and three source candidates (NGC 6541, NGC 6752, and M15) are ranked high, i.e. at the top 23 in the ranking. Only NGC 6652 gave a relatively low expected $\gamma$-ray flux. Among the top 20, we identify the following GCs that have not been reported as $\gamma$-ray source or source candidate before (in descending order of expected $\gamma$-ray flux): Liller 1, M22, NGC 2808, NGC 362, NGC 6540, NGC 1851, Terzan 6, M80, and NGC 6397. On the other hand, nearby $\gamma$-ray emission has been reported for NGC 6441 and NGC 6624 (Abdo et al. 2010c). In this work, we searched for $\gamma$-rays from the GCs in the above list, as well as looked deeper into the cases of NGC 6441, NGC 6541, NGC 6624, and NGC 6752. NGC 6139 (ranked 30th) was also studied.

The $\gamma$-ray data used in this work were obtained between 2008 August 4 and 2010 August 21. We used the Fermi Science Tools v9r15p2 package to reduce and analyze the data provided by the Fermi Science Support Center2. Only those data that passed the most stringent photon selection criteria (i.e. the "diffuse" class) were used. To reduce the contamination from Earth albedo $\gamma$-rays, we excluded events with zenith angles greater than $105^{\circ}$. The instrument response functions (IRFs) "P6_V3_DIFFUSE" were used.

\footnotetext{
${ }^{2}$ http://fermi.gsfc.nasa.gov/ssc/data/analysis/scitools/
} 


\section{3. data analysis and results}

Photons with energies between $E_{\min }$ and $E_{\max }$ that come from a circular region-ofinterest (ROI) around the GCs were included in the analysis. The ROI, as well as $E_{\min }$ and $E_{\max }$, for those GC coincident with $\gamma$-ray emission are given in Table 1. We subtracted the background contribution from all sources in the first Fermi/LAT catalog (1FGL; Abdo et al. 2010b) within the circular region of $15^{\circ}$ radius around the GC position, as well as diffuse emission by including Galactic diffuse model (gll_iem_v02.fit) and isotropic background (isotropic_iem_v02.txt). We assumed single power laws for all first Fermi/LAT catalog sources considered, except for $\gamma$-ray pulsars of which the spectra follow power laws with exponential cut-off (Abdo et al. 2010a, d).

We created the "Test-statistic (TS; Mattox et al. 1996) maps" in the neighboring region of the clusters using the tool gttsmap. These TS maps are created by moving a putative point source through a grid of locations on the sky and maximizing - log(likelihood) at each grid point, while stronger and presumably well-identified sources are included in each fit. Weaker sources would be identified in these maps. Such TS maps around Liller 1, M80, NGC 6139, NGC 6441, NGC 6541, NGC 6624, and NGC 6752 are shown in Figs. 1 to 4 centered on the best-fit centroid of the $\gamma$-ray emission (to be determined in the following). Photons with energy between $E_{\min }$ and $E_{\max }$ as stated in Table 1 were used in generating the maps, except for Liller 1 whose map was generated using photons with energy between 2-30 GeV to avoid contamination from the strong Galactic background due to its Galactic position of $l=354.84$ and $b=-0^{\circ} .16$.

We found evidence for $\gamma$-ray emission within the tidal radius of Liller 1, M80, NGC 6139, NGC 6541, NGC 6624, and NGC 6752. In addition, we found significant $\gamma$-ray emission next to NGC 6441, previously reported in Abdo et al. (2010c). In all these cases, a $\gamma$-ray source candidate in the neighborhood of the corresponding GCs is detected above the modeled background emission. We did not find significant $\gamma$-ray emission within the tidal radius of other candidates: M22, NGC 2808, NGC 362, NGC 6540, NGC 1851, Terzan 6, and NGC 6397.

The TS values and the corresponding significances $(=\sqrt{T S})$ of the $\gamma$-ray emission coincident with the GCs are shown in Table2. The number of gamma-ray photons associated with the corresponding $\gamma$-ray source in the best-fit model provided by the likelihood tool gtlike are also listed. Since the significance values for a number of positions were calculated, this significance has to be corrected according to a "trial factor" to give a "post-trial" significance. This trial factor accounts for the increased probability of finding a fake signal with a number of search positions. We estimate the trial factor as $N_{\mathrm{GC}} \times N_{\mathrm{bin}}$, where $N_{\mathrm{GC}}$ is the number of GCs searched, and $N_{\text {bin }}$ the number of bins within the tidal radius of each GC. We have 
searched for $\gamma$-rays from 30 GCs(including those with the highest encounter rate normalized by its distance and those first mentioned in Abdo et al. 2010c), therefore $N_{\mathrm{GC}}=30$. For $N_{\text {bin }}$, the number of bins is obtained by dividing the area of the tidal radius of an GC (we take the tidal radius of Liller $1,13^{\prime}$, as the averaged tidal radius) by the area of each bin $(0.1 \times 0.1=0.01$ squared degrees $)$. This gives $N_{\text {bin }} \sim 15$. After accounting for the trial factor, the "post-trial" detection significance values are obtained. Since the TS value for adjacent grid points are correlated in the likelihood analysis procedure, the post-trial significance can be considered as conservative estimates.

We then used gtfindsrc to determine the positions of the $\gamma$-ray source candidates and gtlike (unbinned likelihood analysis) to obtain their spectra and their TS values. The integrated photon fluxes and energy fluxes are given for the energy range $100 \mathrm{MeV}$ to $100 \mathrm{GeV}$, using simple extrapolation from the respective models. For the three cases of most significant detection (i.e., Liller 1, NGC 6624, and the $\gamma$-ray source next to NGC 6441), photons between $300 \mathrm{MeV}$ and $100 \mathrm{GeV}$ are divided into 6 logarithmically-equally spaced energy bins. The flux in each bin was obtained by gtlike using photons in the corresponding bin, while both the normalization and photon indices of all $\gamma$-ray sources and the diffuse background components, namely Galactic diffuse and isotropic components, were set to be free. Only for cases where the photon statistics is not enough for a satisfactory fit, the photon index was fixed at the best-fit value shown in Table 2 .

We also performed a long-term temporal analysis of the $7 \gamma$-ray sources, in which the 2year data were binned into several periods depending on the photon statistics. No significant

$\gamma$-ray variability nor flaring period was found, indicating that the sources are stable in radiating $\gamma$-rays.

In the following section, we discuss the results of each of the GCs in more details.

\section{Gamma-ray properties from individual clusters}

\subsection{Gamma-ray emission within cluster's tidal radius}

\subsubsection{Liller 1}

The encounter rate of Liller 1 is the highest among the target list mentioned in Section 2 . As seen in Fig. 1, $\gamma$-ray emission from the direction of Liller 1 was found. Fitting the photon spectrum $(d N / d E)$ with the single power law, the TS value of this source is 107 , corresponding to a detection significance of $10.3 \sigma$ (i.e. $9.4 \sigma$ post-trial). The best-fit position is R.A. $=263.20$, Dec. $=-33.39 \pm 0.04$ (stat) $(\mathrm{J} 2000 ; 1-\sigma$ uncertainty). For localization of 
a typical $\gamma$-ray source, the systematic position error is estimated to be about $40 \%$ of the statistical error (Abdo et al. 2009c) 3. The best-fit position is 7'.5 from the core position of the GC Liller 1, which is within its tidal radius (12.57). Fig. 5 depicts the spectrum (in $E^{2} d N / d E$ ) of the $\gamma$-ray emission between $300 \mathrm{MeV}$ and $100 \mathrm{GeV}$. The photon spectrum of Liller 1 can be well fit by a single power law with an index of $2.2 \pm 0.1$, and the integral photon flux between $100 \mathrm{MeV}$ and $100 \mathrm{GeV}$ is $F_{0.1-100 \mathrm{GeV}}=(6.9 \pm 2.3) \times 10^{-8} \mathrm{~cm}^{-2} \mathrm{~s}^{-1}$ in this model. On the other hand, the parameters provided by the power law with an exponential cut-off (PLE) model with all three parameters being free are not well constrained; we then fixed the cut-off energy in this model at $2 \mathrm{GeV}$ and $10 \mathrm{GeV}$, and obtained smaller values of TS (i.e. 90 and 100, respectively). We therefore do not consider this model to be significant. We found that the rightmost spectral point shown in Fig. 5, that corresponds to 40-100 GeV emission, were detected with a TS value of 16 , corresponding to a significance of $4 \sigma$. At a distance of $9.6 \mathrm{kpc}$ Harris 1996, 2003 version; which is within the range $(8.3 \pm 1.8) \mathrm{kpc}$ given in Ortolani et al. (2007)), the $\gamma$-ray luminosity of Liller 1 is $L_{0.1-100 \mathrm{GeV}}=$ $(5.9 \pm 2.0) \times 10^{35} \mathrm{erg} \mathrm{s}^{-1}$, which is the largest among all detected $\gamma$-ray emitting GCs by now.

\subsection{2. $M 80$}

Among the GCs in the target list, M80 was found to be a possibly $\gamma$-ray emitting GC. This GC is located at the Galactic coordinate of $(l, b)=\left(352^{\circ} 6,19{ }^{\circ}\right)$, i.e. well outside the Galactic plane. Therefore we extended the ROI radius to $10^{\circ}$ and included photons with energies $200 \mathrm{MeV}$ to $100 \mathrm{GeV}$ in the analysis. Inside the tidal radius of M80 we found evidence for $\gamma$-ray emission that peaks at a TS value of 27 , corresponding to a significance of $5.2 \sigma$ (i.e. $3.9 \sigma$ post-trial), as shown in Fig. 1 (region $A$ ). We therefore regard M80 as a possible $\gamma$-ray emitter. In addition, it is possible that the possible $\gamma$-ray emission region may extend well outside the GC, i.e. into region $B$. Formally we cannot separate the whole $\gamma$ ray emission into two emitting regions. Assuming that the $\gamma$-ray emitting region coincident with the GC (i.e. region $A$ ) is a separate source, its best-fit position is R.A. $=244.23$, Dec. $=-23.02 \pm 0.06$ (stat) (J2000). Its distance of 3'3 from the GC core position makes the two positions consistent within errors. The photon spectrum of this $\gamma$-ray source can be well fit by a single power law with an index of $2.0 \pm 0.2$. The integral $\gamma$-ray flux is $F_{0.1-100 \mathrm{GeV}}=(5.5 \pm 3.6) \times 10^{-9} \mathrm{~cm}^{-2} \mathrm{~s}^{-1}$. The low statistics of photons does not allow for accurate determination of the parameter values in the PLE model; we therefore do not consider this model. At a distance of $10.3_{-0.7}^{+0.8} \mathrm{kpc}$ (Brocato et al. 1998), $L_{0.1-100 \mathrm{GeV}}=$ $8.4_{-5.8}^{+7.6} \times 10^{34} \mathrm{erg} \mathrm{s}^{-1}$.

\footnotetext{
${ }^{3}$ We assume similar systematic errors for other GCs as well.
} 
The best-fit position of the $\gamma$-ray emitting region $B(\mathrm{TS}=30)$ is R.A. $=244.42$, Dec. $=$ $-23.50 \pm 0.07$ (stat) (J2000). The photon spectrum of this $\gamma$-ray source can be fit by a single power law with an index of $2.2 \pm 0.2$. The integral $\gamma$-ray flux is $F_{0.1-100 \mathrm{GeV}}=(1.1 \pm 0.6) \times$ $10^{-8} \mathrm{~cm}^{-2} \mathrm{~s}^{-1}$.

\subsection{3. $N G C 6139$}

The encounter rate of NGC 6139 is the 30th highest in our target list. We found evidence for $\gamma$-ray emission at the position of NGC 6139. The TS value of this source candidate is 32 , corresponding to a detection significance of $5.6 \sigma$ (i.e. $4.5 \sigma$ post-trial), obtained in the power law model. We therefore regard this detection as a marginal detection. The TS map around NGC 6139 is shown in Fig. 2, The best-fit position is R.A. $=246.83$, Dec. $=-38.90$ \pm 0 .07(stat) (J2000), which is 5'.1 from the core position of NGC 6139, but within its tidal radius (8.52). If the $\gamma$-ray source is confirmed, the $\gamma$-ray spectrum of NGC 6139 can be well fit by a single power law with an index of $2.1 \pm 0.2$. The integrated photon flux between $100 \mathrm{MeV}$ and $100 \mathrm{GeV}$ is $F_{0.1-100 \mathrm{GeV}}=(9.9 \pm 5.4) \times 10^{-9} \mathrm{~cm}^{-2} \mathrm{~s}^{-1}$. The low statistics of photons does not allow for accurate determination of the parameter values in the PLE model; we therefore do not consider this model. At a distance of $10.1 \mathrm{kpc}$ (Harris 1996, 2003 version), the $\gamma$-ray luminosity of NGC 6139 is $L_{0.1-100 \mathrm{GeV}}=(1.1 \pm 0.6) \times 10^{35} \mathrm{erg} \mathrm{s}^{-1}$.

\subsubsection{NGC 6541}

Inside the tidal radius of NGC 6541, we found a possible $\gamma$-ray source that peaks at $\mathrm{TS}=19$, corresponding to a significance of $4.4 \sigma$ (i.e. $2.8 \sigma$ post-trial). Therefore, we consider

this source to be possibly detected, consistent with the finding of Abdo et al. (2010c). The TS map is shown in Fig. 2. The best-fit position is R.A. $=272.04$, Dec. $=-43.85 \pm 0.11$ (stat) (J2000), 8'.9 from the core position of the GC NGC 6541, well within its tidal radius (29'.60). This position is consistent with those derived in Abdo et al. (2010b, c). Therefore, we identify this source candidate to be 1FGL J1807.6-4341. A power-law fit of the photon spectrum gave an index of $2.2 \pm 0.2$ and an integrated photon flux of $F_{0.1-100 \mathrm{GeV}}=(9.5 \pm 4.4) \times 10^{-9} \mathrm{~cm}^{-2} \mathrm{~s}^{-1}$. This flux is consistent with the upper limit of $1.1 \times 10^{-8} \mathrm{~cm}^{-2} \mathrm{~s}^{-1}$ as given in Abdo et al. (2010c), noting that their flux was calculated using the PLE model. Moreover, our derived integrated energy flux of $E_{0.1-100 \mathrm{GeV}}=(6.5 \pm 3.0) \times 10^{-12} \mathrm{erg} \mathrm{cm}^{-1} \mathrm{~s}^{-1}$ are consistent with that given in Abdo et al. (2010b). The PLE model was not used in the likelihood fit because of the putative nature of the $\gamma$-ray emission. At a distance of $(6.9 \pm 0.7) \mathrm{kpc}$ (Lee \& Carney 2006), the $\gamma$-ray luminosity of the $\gamma$-ray source candidate is $L_{0.1-100 \mathrm{GeV}}=3.7_{-2.1}^{+2.9} \times 10^{34} \mathrm{erg} \mathrm{s}^{-1}$. 


\subsubsection{NGC 6624}

The $\gamma$-ray source 1FGL J1823.4-3009 lies close to NGC 6624 (Abdo et al. 2010b. c). Using a larger data set, we confirmed this $\gamma$-ray source and obtained a TS value of 121 , corresponding to a detection significance of $11.0 \sigma$ (i.e. $10.1 \sigma$ post-trial). The best-fit position was found to be R.A. $=275 \circ 86$, Dec. $=-30.16 \pm 0.05$ (stat) $(\mathrm{J} 2000), 12$ '.2 from the core position of NGC 6624 (shown in Fig. 3). This position is consistent with that reported in Abdo et al. (2010c) and it lies within the tidal radius of the cluster (20'55). We obtained a single power-law fit of the photon spectrum with an index $2.2 \pm 0.1$, in which the integrated $\gamma$-ray flux is $F_{0.1-100 \mathrm{GeV}}=(2.6 \pm 0.6) \times 10^{-8} \mathrm{~cm}^{-2} \mathrm{~s}^{-1}$. The above power-law index and our derived integrated energy flux of $E_{0.1-100 \mathrm{GeV}}=(2.0 \pm 0.5) \times 10^{-11} \mathrm{erg} \mathrm{cm}^{-1} \mathrm{~s}^{-1}$ are consistent with that given in Abdo et al. (2010b). On the other hand, the parameters provided by the PLE model (with all three parameters free) are not well constrained; we then fixed the cut-off energy in this model at $600 \mathrm{MeV}, 1 \mathrm{GeV}, 1.5 \mathrm{GeV}, 2 \mathrm{GeV}, 3 \mathrm{GeV}$ and $5 \mathrm{GeV}$, and obtained TS values of $111,120,121,120,118$, and 116, respectively). Fixing the cutoff energy at $1.5 \mathrm{GeV}$, the power law index was found to be $0.9 \pm 0.2$, and the integrated $\gamma$-ray flux was found to be $F_{0.1-100 \mathrm{GeV}}=1.0_{-0.5}^{+0.7} \times 10^{-8} \mathrm{~cm}^{-2} \mathrm{~s}^{-1}$. Since this model gives the same $\mathrm{TS}(=121)$ as the power law, it is a model as good as the power-law model. We found that the two rightmost spectral points shown in Fig. 6. that together correspond to $14-100 \mathrm{GeV}$ emission, were detected with a TS value of $\sim 10$ each, corresponding to a significance of $3 \sigma$ each. At a distance of $7.9 \mathrm{kpc}$ (Harris 1996, 2003 version), the $\gamma$-ray luminosity of NGC 6624 (using the power-law model) is $L_{0.1-100 \mathrm{GeV}}=(1.5 \pm 0.3) \times 10^{35} \mathrm{erg} \mathrm{s}^{-1}$.

\subsubsection{NGC 6752}

We found significant $\gamma$-ray emission from NGC 6752. This source has a TS value of 49, corresponding to a significance of $7.0 \sigma$ (i.e. $6.0 \sigma$ post-trial), in the power law model. As shown in Fig. 3, the source is compact and located close to the core of NGC 6752. The best-fit position is R.A. $=287.57$, Dec. $=-59.96 \pm 0.09$ (stat) $(\mathrm{J} 2000), 4^{\prime} .7$ from the core position of NGC 6752, and is consistent with being emitted from within the half-mass radius, as shown in Fig. 3. This position is consistent with that derived in Abdo et al. (2010c). The photon spectrum of the source can be well fit by a single power law with an index of $2.0 \pm 0.2$. The integrated photon flux between $100 \mathrm{MeV}$ and $100 \mathrm{GeV}$ is $F_{0.1-100 \mathrm{GeV}}=$ $(6.0 \pm 2.8) \times 10^{-9} \mathrm{~cm}^{-2} \mathrm{~s}^{-1}$. This flux is consistent with the upper limit of $7 \times 10^{-9} \mathrm{~cm}^{-2} \mathrm{~s}^{-1}$ as given in Abdo et al. (2010c), noting that their flux was calculated using the PLE model. The parameters provided by the PLE model (with all three parameters free) are not well constrained; we then fixed the cut-off energy at $600 \mathrm{MeV}, 1 \mathrm{GeV}, 3 \mathrm{GeV}$ and $10 \mathrm{GeV}$, and 
obtained smaller values of TS (i.e. 23, 27, 34, and 42, respectively). We therefore do not consider this model to be significant. At a distance of $(4.4 \pm 0.1) \mathrm{kpc}$ (Harris 1996, 2003 version), the $\gamma$-ray luminosity of NGC 6752 is $L_{0.1-100 \mathrm{GeV}}=(1.4 \pm 0.7) \times 10^{34} \mathrm{erg} \mathrm{s}^{-1}$.

\subsection{Gamma-ray emission outside cluster's tidal radius: the case of NGC 6441}

A $\gamma$-ray source next to NGC 6441 was first reported by Abdo et al. (2010c). Using a larger data set, we also found a source (a TS value of 101 is obtained with the PLE model, corresponding to a detection significance of $10.0 \sigma$, i.e. $9.1 \sigma$ post-trial) with the best-fit position of R.A. $=267^{\circ} 63$, Dec. $=-36^{\circ} 89 \pm 0.09$ (stat) $(\mathrm{J} 2000), 10^{\prime} 6$ from the position of NGC 6441 (shown in Fig. (4). This position is consistent with that reported in Abdo et al. (2010c) and it likely lies outside the tidal radius of the cluster (8'00) but it is still possible that some emission is from within the tidal radius given the current statistical and systematic errors. We obtained a single power-law fit with an index $2.4 \pm 0.1$, in which the integrated $\gamma$ ray flux is $F_{0.1-100 \mathrm{GeV}}=(4.1 \pm 1.0) \times 10^{-8} \mathrm{~cm}^{-2} \mathrm{~s}^{-1}$. This fit gives a TS value of 73 . The photon spectrum can better be fit by a PLE where the photon index, $\Gamma$, is $0.4 \pm 0.9$ and the cutoff energy is $(1.0 \pm 0.5) \mathrm{GeV}$, giving $T S=100$. To further constrain the spectral parameters, we proceeded to fix $\Gamma$ at 0.2 to 2.0 (with steps of 0.2 ) while letting the normalization and $E_{\mathrm{c}}$ free, and found that TS $=101$ for $\Gamma_{\gamma}=0.2,0.4$, and 0.6 and the TS value decreases with increasing $\Gamma$. If one fixes $\Gamma_{\gamma}$ at $0.4, E_{\mathrm{c}}=(1.0 \pm 0.1) \mathrm{GeV}$ is obtained. In this case, the integrated $\gamma$-ray flux is $F_{0.1-100 \mathrm{GeV}}=(1.0 \pm 0.2) \times 10^{-8} \mathrm{~cm}^{-2} \mathrm{~s}^{-1}$. The significance of the PLE model over the single power law model can be estimated by $T S_{\mathrm{PLE}}-T S_{\mathrm{PL}}=28$, which corresponds to $5.3 \sigma$. Therefore, we consider the PLE model to be a significantly better model than the power law model. The PLE model spectrum, as well as the data binned in different energies, is depicted in Fig. 7. At a distance of $11.7 \mathrm{kpc}$ (Valenti et al. 2004), the $\gamma$-ray luminosity of NGC 6441 (using the power law model) is $L_{0.1-100 \mathrm{GeV}}=(3.7 \pm 0.9) \times 10^{35} \mathrm{erg} \mathrm{s}^{-1}$, or $L_{0.1-100 \mathrm{GeV}}=(1.3 \pm 0.2) \times 10^{35} \mathrm{erg} \mathrm{s}^{-1}$ (using the PLE model).

\section{5. discussion}

In this work, we report the discovery of three new $\gamma$-ray emitting GCs: Liller 1 , NGC 6624, and NGC 6752, and we found evidence for $\gamma$-ray emission from M80, NGC 6139, and NGC 6541. We also confirmed the $\gamma$-ray detection next to NGC 6441 first mentioned

by Abdo et al. (2010c). We searched through various X-ray and radio catalogs for plausible $\gamma$-ray sources in the neighborhood of the GCs. We found two pulsars next to Liller 1, as shown in Fig. 1 (left panel). The spin-down luminosity of PSR J1733-3322 and PSR J1734- 
3333 , is $8.3 \times 10^{31} \mathrm{erg} \mathrm{s}^{-1}$ and $5.6 \times 10^{34} \mathrm{erg} \mathrm{s}^{-1}$, respectively (Morris et al. 2002), i.e. more than an order of magnitude lower than the observed $\gamma$-ray luminosity of $10^{36} \mathrm{erg} \mathrm{s}^{-1}$. Together with their large offsets from the $\gamma$-ray emitting region, the association of the observed $\gamma$-rays with either pulsar is unlikely. In all cases, the GCs seem to be the only plausible counterparts of the observed $\gamma$-rays (we refer to region A in the case of M80). Without clear counterparts in other wavelengths, the nature of the $\gamma$-ray emitting region $B$ shown in Fig. 1 (right panel) remains unclear, and its relation with M80 cannot be confirmed or ruled out at this stage.

Several MSPs are known in NGC 6441, NGC 6624, and NGC 6752 (all located close to its core) but none has yet been uncovered in other cases (e.g., Hui et al. 2009). We speculate that there is a population of MSPs - the only known steady $\gamma$-ray emitters in GCs - in the $\gamma$-ray emitting GCs that we have reported here. For example, the brighter X-ray population of M80 is in many ways similar to 47 Tucanae, the first $\gamma$-ray GC known, while the fainter X-ray sources may differ (Heinke et al. 2003). The discovery of $\gamma$-rays from M80, if confirmed, would suggest that it also hosts a population of MSPs. Under the assumption that the observed $\gamma$-ray luminosity depends solely on the number of MSPs (e.g. in the magnetospheric model), the predicted number of MSPs in the cluster using Eq.(1) of Abdo et al. (2010c) is listed in Table 2. Future multiwavelength observations of any MSPs should help in understanding the $\gamma$-ray production mechanisms and the underlying MSP populations.

The $\gamma$-ray spectra are in general consistent with single power law model, and shows no convincing evidence for cut-off at high energies. It is mainly due to limited photon statistics. However, the cases of the more significant cases of Liller 1 and NGC 6624, in which $\gamma$-ray are found within the tidal radius, suggests that $>10 \mathrm{GeV}$ emission exists at least for these two cases. If this is true, this high energy emission conform with the IC model, rather than the magnetospheric model. The $>10 \mathrm{GeV}$ emission may correspond to the IC peak as predicted in Bednarek \& Sitarek (2007) and Cheng et al. (2010).

If the $\gamma$-ray emission is associated with the GCs, one striking feature is the displacement of the $\gamma$-ray emission from the GC cores. We briefly discuss this feature in the context of two radiation scenarios commonly discussed in the literature.

In the pulsar magnetosphere model, one expects the emission to coincide spatially with the MSP population. While MSPs should concentrate in the GC core in general, the finite number of MSPs in a GC (i.e., 10, see Table 2, except for Liller 1) may invoke some offset of the MSPs - and thus the $\gamma$-rays they emit - from the core. Furthermore, in addition to the MSP population formed in the core, some MSPs are formed near the tidal radius of the GCs. The superposition of $\gamma$-rays from these two populations may not coincide with the 
$\mathrm{GC}$ core, but is at a certain distance from the core within the tidal radius. However, this scenario cannot accommodate the case of the $\gamma$-ray source next to NGC 6441.

In the inverse Compton models, the $\gamma$-ray emitting region depends not only on the distribution of the underlying MSPs, but also on the cooling timescale and the diffusion timescale of the accelerated particles. When the diffusion timescale is much smaller than the cooling timescale, the $\gamma$-rays may come from the outskirts of the GCs.

In the cases of Liller 1 and NGC 6624, the gamma-ray spectrum (cf. Fig. [5] and Fig. 6) can extend up to energies $>40 \mathrm{GeV}$, which cannot be explained in terms of curvature radiation inside the light cylinder. Recently, Cheng et al. (2010) have suggested that inverse Compton scattering between background relativistic electrons/positrons in pulsar wind and the soft photons from the galactic disk can produce the observed gamma-rays from globular clusters. They predict that if the inverse Compton upscattered Galactic infrared photons are responsible for $\mathrm{GeV}$ photons, then the Compton upscattered galactic optical photons can produce gamma-rays up to $100 \mathrm{GeV}$. They also predict that the gamma-rays are diffuse and are emitted from region much beyond the core of the globular cluster. If this is true the center of gamma-ray emission regions is affected by three factors: the proper motion of the globular cluster, the asymmetric diffusion coefficient and nearby optical/IR external sources like stars and nearby regions containing an enhanced amount of dust.

The discovery of several more $\gamma$-ray emitting GCs reported in this work has allowed population studies to be carried out in order to better understand the relationship between the $\gamma$-ray properties of GCs and other parameters of GCs. Abdo et al. (2010c) found that the stellar encounter rate scales with the $\gamma$-ray luminosity, based on a sample of $8 \gamma$-ray emitting GCs. While the $\gamma$-ray luminosity should correlate with the number of MSPs in the clusters in both models, it is expected that the low-energy photon energy density is also a good estimator of $\gamma$-rays in the IC models. Recently, using a total of 15 GCs that are $\gamma$-ray sources or source candidates, including those reported in this work and those previously known (Abdo et al. 2010c), Hui et al. (2011) have shown that correlations also exist between $\gamma$-ray luminosity and metallicity, as well as $\gamma$-ray luminosity and optical/infrared photon energy density.

To conclude, we report the detection of new $\gamma$-ray emitting GCs, based on a ranking list that replies on the encounter rate of the GCs. The $\gamma$-ray luminosity of Liller 1 is the highest of all known $\gamma$-ray GCs. Its non-detection before this work may be due to its position that lies on the Galactic plane, where Galactic diffuse emission is very strong. Liller 1 has very high encounter rate and metallicity, and may indeed host a large population of MSPs that have not been uncovered. Some $\gamma$-ray emitting GCs presented here are at distances of $\sim 10$ kpc away from us, whereas Abdo et al. (2010b) present the upper limits of known GCs out to a distance of $6 \mathrm{kpc}$ only. Therefore, we have demonstrated that the study here has 
gone a further step towards finding new sources even at larger distances. This then helps to expand the sample of known $\gamma$-ray GCs for further studies.

We acknowledge the use of data and software facilities from the FSSC, managed by the HEASARC at the Goddard Space Flight Center. CYH is supported by research fund of Chungnam National University in 2010. KSC is supported by a GRF grant of Hong Kong Government under HKU700908P, and AKHK is supported partly by the National Science Council of the Republic of China (Taiwan) through grant NSC96-2112-M-007-037-MY3 and NSC99-2112-M-007-004-MY3.

\section{REFERENCES}

Abdo, A. A., et al. (Fermi/LAT Collaboration) 2009a, Science, 325, 845

Abdo, A. A., et al. (Fermi/LAT Collaboration) 2009b, Science, 325, 848

Abdo, A. A., Ackermann, M., Ajello, M., et al. (Fermi/LAT Collaboration) 2009c, ApJS, 183,46

Abdo, A. A., et al. (Fermi/LAT Collaboration) 2010a, ApJS, 187, 460

Abdo, A. A., et al. (Fermi/LAT Collaboration) 2010b, ApJS, 188, 405

Abdo, A. A., et al. (Fermi/LAT Collaboration) 2010c, A\&A, 524, 75

Aharonian, F., et al. (HESS Collaboration) 2009, A\&A, 499, 273

Anderhub, H., et al. (MAGIC Collaboration) 2009, ApJ, 702, 266

Atwood, W. B., et al. (Fermi/LAT Collaboration) 2009, ApJ, 697, 1071

Bednarek, W., \& Sitarek, J. 2007, MNRAS, 377, 920

Brocato, E., Castellani, V., Scotti, G. A., Saviane, I., Piotto, G., \& Ferraro, F. R. 1998, A\&A, 335, 929

Cheng, K. S., Chernyshov, D. O., Dogiel, V. A., Hui, C. Y., \& Kong, A. K. H. 2010, ApJ, 723,1219

Gendre, B., Barret, D., \& Webb, N. 2003, A\&A, 403, L11 
Gnedin, O. Y., Zhao, H. S., Pringle, J. E., Fall, S. M., Livio, M., \& Meylan, G. 2002, ApJ, 568, L23

Harris, W. E. 1996, AJ, 112, 1487 (2003 version)

Heinke, C. O., Grindlay, J. E., Edmonds, P. D., Lloyd, D. A., Murray, S. S., Cohn, H. N., \& Lugger, P. M. 2003, ApJ, 598, 516

Hui, C. Y., Huang, H. H., Cheng, K. S., Taam, R. E., \& Becker, W. 2009, Astronomical Society of the Pacific Conference Series, 404, 149

Hui, C. Y., Cheng, K. S., \& Taam, R. E. 2010, ApJ, 714, 1149

Hui, C. Y., Cheng, K. S., Wang, Y., Tam, P. H. T., Kong, A. K. H., Chernyshov, D. O., \& Dogiel, V. A. 2011, ApJ, 726, 100

Kong, A. K. H., Hui, C. Y., \& Cheng, K. S. 2010, ApJ, 712, L36

Lee, J. W. \& Carney, B. W. 2006, AJ, 132, 2171

Manchester, R. N., Hobbs, G. B., Teoh, A., \& Hobbs, M. 2005, AJ, 129, 1993

Mattox, J. R., et al. 1996, ApJ, 461, 396

Morris, D. J., et al. 2002, MNRAS, 335, 275

Ortolani, S., Barbuy, B., Bica, E., Zoccali, M., \& Renzini, A. 2007, A\&A, 470, 1043

Pooley, D., et al. 2003, ApJ, 591, L131

Pooley, D., \& Hut, P. 2006, ApJ, 646, L143

Valenti, E., Ferraro, F. R., \& Origlia, L. 2004, MNRAS, 351, 1204

Venter, C., \& de Jager, O. C. 2008, ApJ, 680, L125

Verbunt, F., \& Hut, P. 1987, Proceedings of the IAU Symposium No. 125, The Origin and Evolution of Neutron Stars, 187 
Table 1: Region-of-interest (ROI), minimum photon energy $\left(E_{\min }\right)$, and maximum photon energy $\left(E_{\max }\right)$ in the analysis of the GCs

\begin{tabular}{|c|c|c|c|c|c|}
\hline \multirow{3}{*}{ GC name } & \multicolumn{2}{|c|}{ ROI center } & ROI radius & \multirow[t]{2}{*}{$E_{\min }$} & \multirow[t]{2}{*}{$\overline{E_{\max }}$} \\
\hline & R.A. (J2000) & Dec. (J2000) & & & \\
\hline & $\left({ }^{\circ}\right)$ & $\left({ }^{\circ}\right)$ & $\left(0^{\circ}\right)$ & $(\mathrm{GeV})$ & $(\mathrm{GeV})$ \\
\hline Liller 1 & 263.20 & -33.39 & 5 & 0.3 & 100 \\
\hline M80 & 244.23 & -23.02 & 10 & 0.2 & 100 \\
\hline NGC 6139 & 246.83 & -38.90 & 6 & 0.3 & 100 \\
\hline NGC 6441 & 267.63 & -36.89 & 5 & 0.3 & 100 \\
\hline NGC 6541 & 272.04 & -43.85 & 6 & 0.3 & 100 \\
\hline NGC 6624 & 275.86 & -30.16 & 6 & 0.3 & 100 \\
\hline NGC 6752 & 287.57 & -59.96 & 10 & 0.2 & 100 \\
\hline
\end{tabular}


Table 2: Summary of $\gamma$-ray results of the GCs

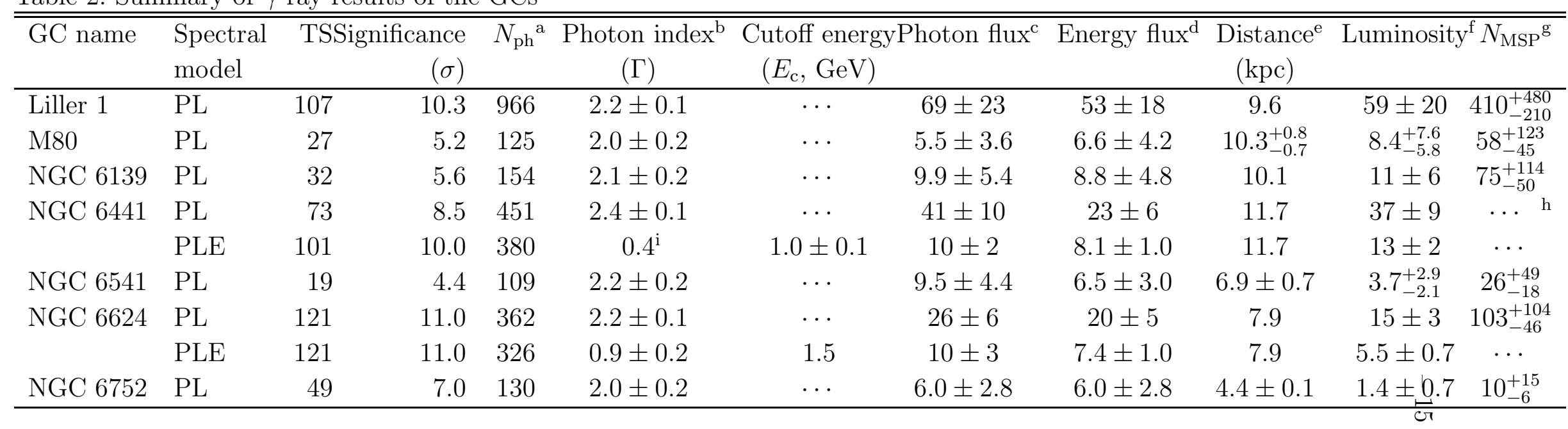

${ }^{a}$ Modeled photon number associated with the corresponding $\gamma$-ray source in the best likelihood fit

${ }^{b}$ All the quoted errors are statistical and $1 \sigma$ for one parameter of interest.

${ }^{c}$ Integrated $0.1-100 \mathrm{GeV}$ photon flux in unit of $10^{-9} \mathrm{~cm}^{-2} \mathrm{~s}^{-1}$

${ }^{d}$ Integrated $0.1-100 \mathrm{GeV}$ energy flux in unit of $10^{-12} \mathrm{erg} \mathrm{cm}^{-2} \mathrm{~s}^{-1}$

${ }^{e}$ Distance adopted from Harris (1996, 2003 version), except for M80 (Brocato et al. 1998), NGC 6541 (Lee \& Carnev 2006), and NGC 6441 (Valenti et al. 2004).

${ }^{f} 0.1-100 \mathrm{GeV}$ luminosity in unit of $10^{34} \mathrm{erg} \mathrm{s}^{-1}$

${ }^{g}$ Predicted number of MSPs in the cluster using Eq.(1) of Abdo et al. (2010c), under the assumption that the observed $\gamma$-ray luminosity depends solely on the number of MSPs

${ }^{h}$ In this case, it is unlikely that the number of MSPs is the sole factor in determining the observed $\gamma$-ray luminosity due to the offset

${ }^{i}$ Model parameters without quoted errors are fixed at the value given. 

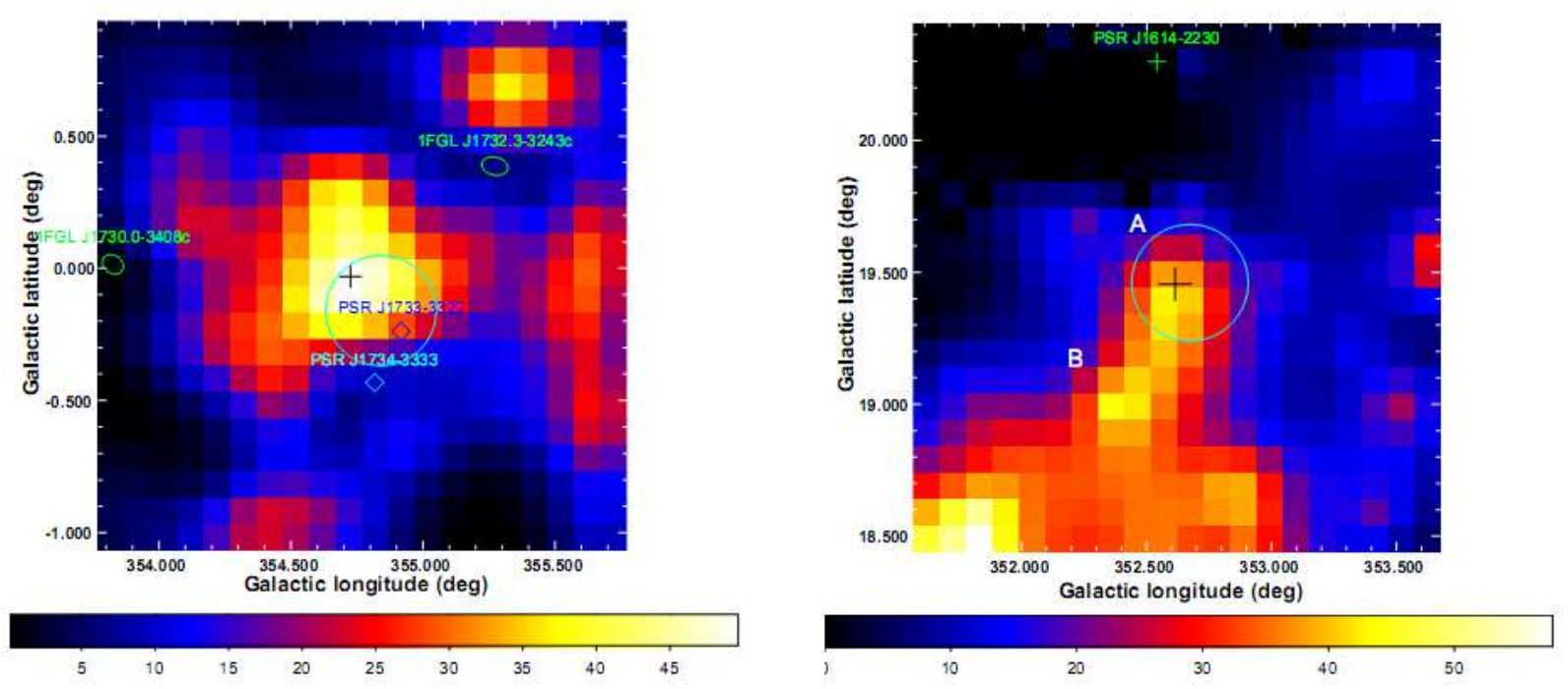

Fig. 1. - The Test-statistic (TS) maps of regions of $2^{\circ} \times 2^{\circ}$ centered on the best-fit centroids (labeled by crosses) of the $\gamma$-ray emission from Liller 1 (left) and M80 (right). The size of each cross indicates the 1- $\sigma$ statistical error in the determination of the centroid position. The color scale shows the TS value of every bin of an area $0^{\circ} .1 \times 0.1$. The light blue circles represent the tidal radius of the respective GCs compiled in Harris (1996, 2003 version). Left: PSR J1733-3322 and PSR J1734-3333, are marked by two white diamonds. The $68 \%$ error positions of the LAT first catalog sources 1FGL J1732.3-3243c and 1FGL J1730.0-3408c (treated as background) are shown as eclipses. Right: The position of the $\gamma$-ray MSP PSR J1614-2230 (Abdo et al. 2009b) is shown as a cross. 

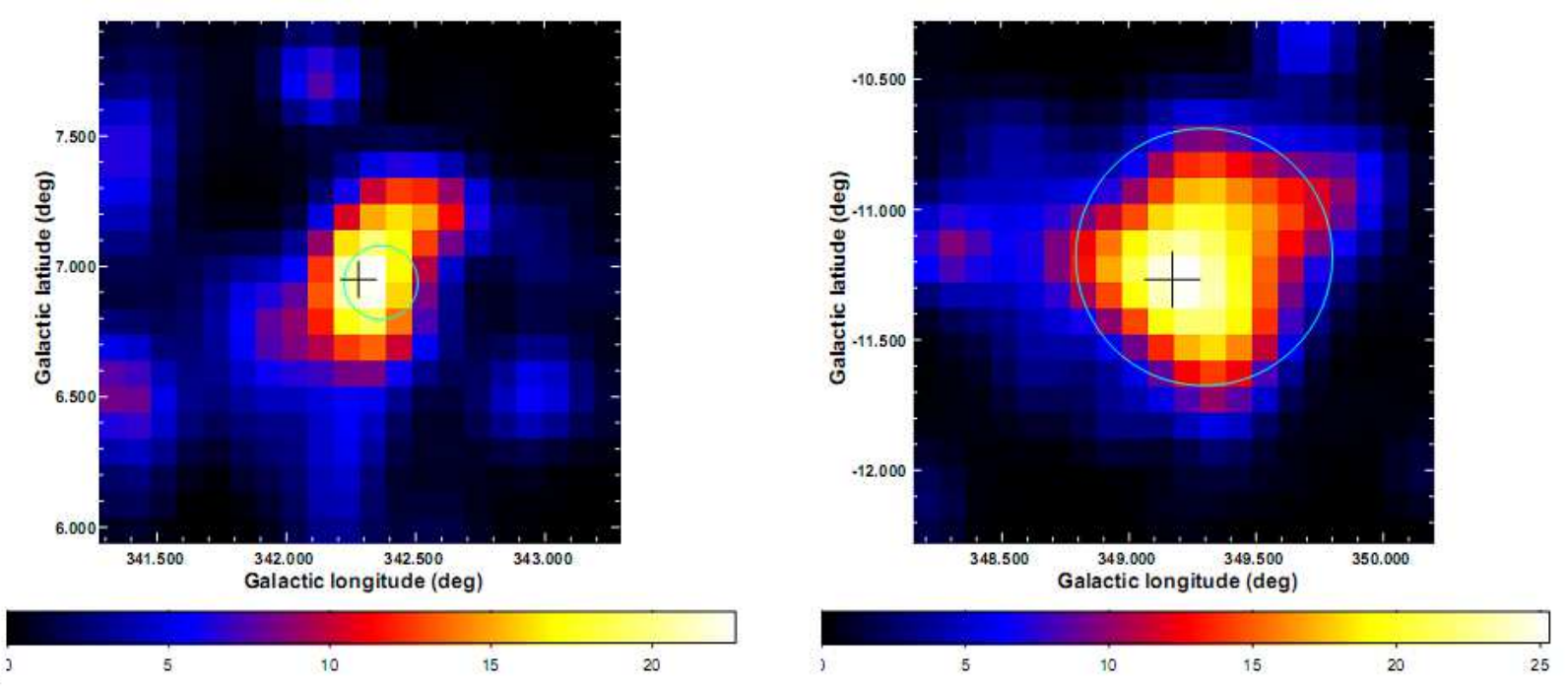

Fig. 2. - The TS maps of regions of $2^{\circ} \times 2^{\circ}$ centered on the best-fit centroids (labeled by crosses) of the $\gamma$-ray emission from NGC 6139 (left) and NGC 6541 (right). The circles represent the tidal radius of NGC 6139 (left) and NGC 6541 (right) (Harris 1996, 2003 version). The meanings of the color scale and the size of the crosses are the same as in Figure 1] 

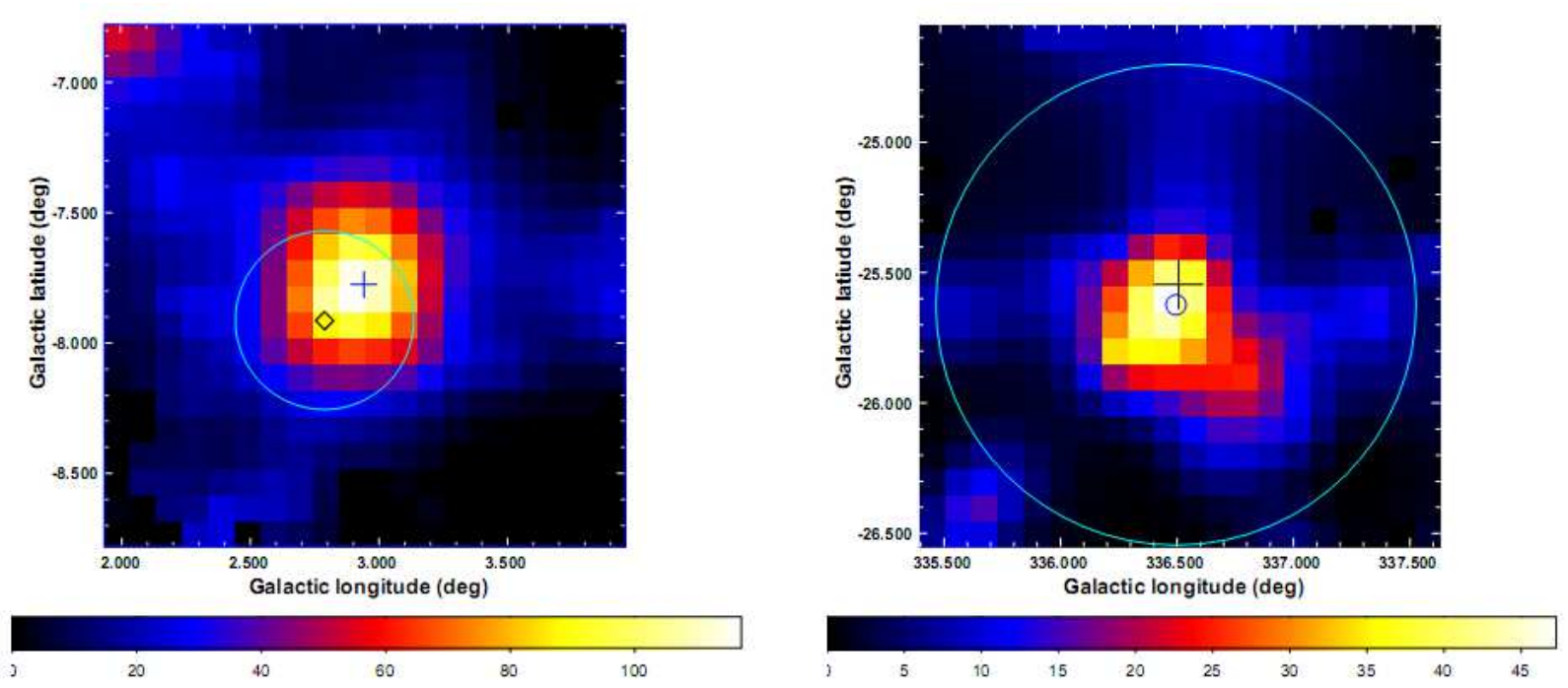

Fig. 3. - The TS maps of regions of $2^{\circ} \times 2^{\circ}$ centered on the best-fit centroids (labeled by crosses) of the $\gamma$-ray emission from NGC 6624 (left) and NGC 6752 (right). The TS map of a region of $2^{\circ} \times 2^{\circ}$ centered on the best-fit centroid (labeled by a cross) of the $\gamma$-ray emission next to NGC 6624. Left panel: The circle represents the tidal radius of NGC 6624 (Harris 1996, 2003 version). The white diamond shows the position of both PSR B1820-30A and PSR B1820-30B. Right panel: The outer and inner circle represents the tidal radius and half-mass radius of NGC 6752, respectively (Harris 1996, 2003 version). The meanings of the color scale and the size of the crosses are the same as in Figure 1. 


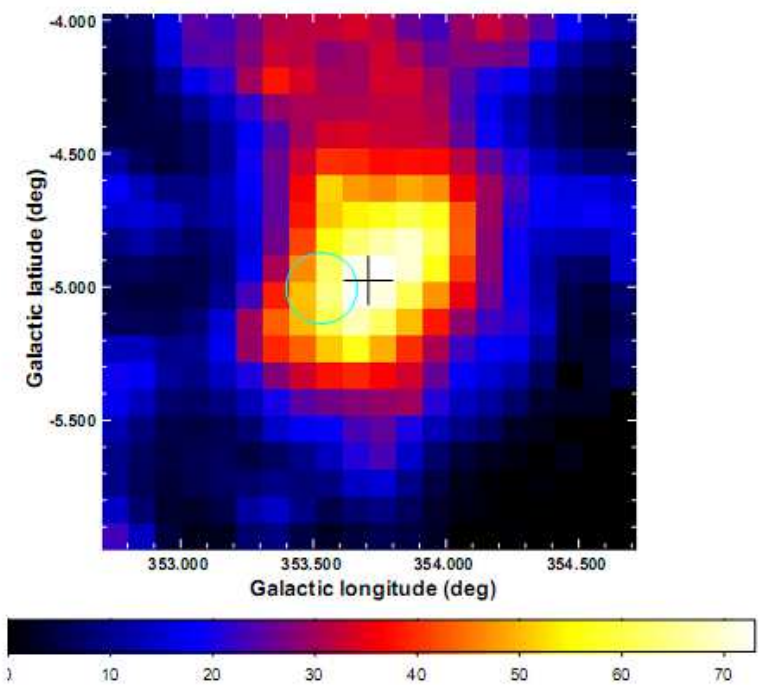

Fig. 4. - The TS map of a region of $2^{\circ} \times 2^{\circ}$ centered on the best-fit centroid (labeled by a cross) of the $\gamma$-ray emission next to NGC 6441. The circle represents the tidal radius of NGC 6441 (Harris 1996, 2003 version). The meanings of the color scale and the size of the cross are the same as in Figure 1 . 


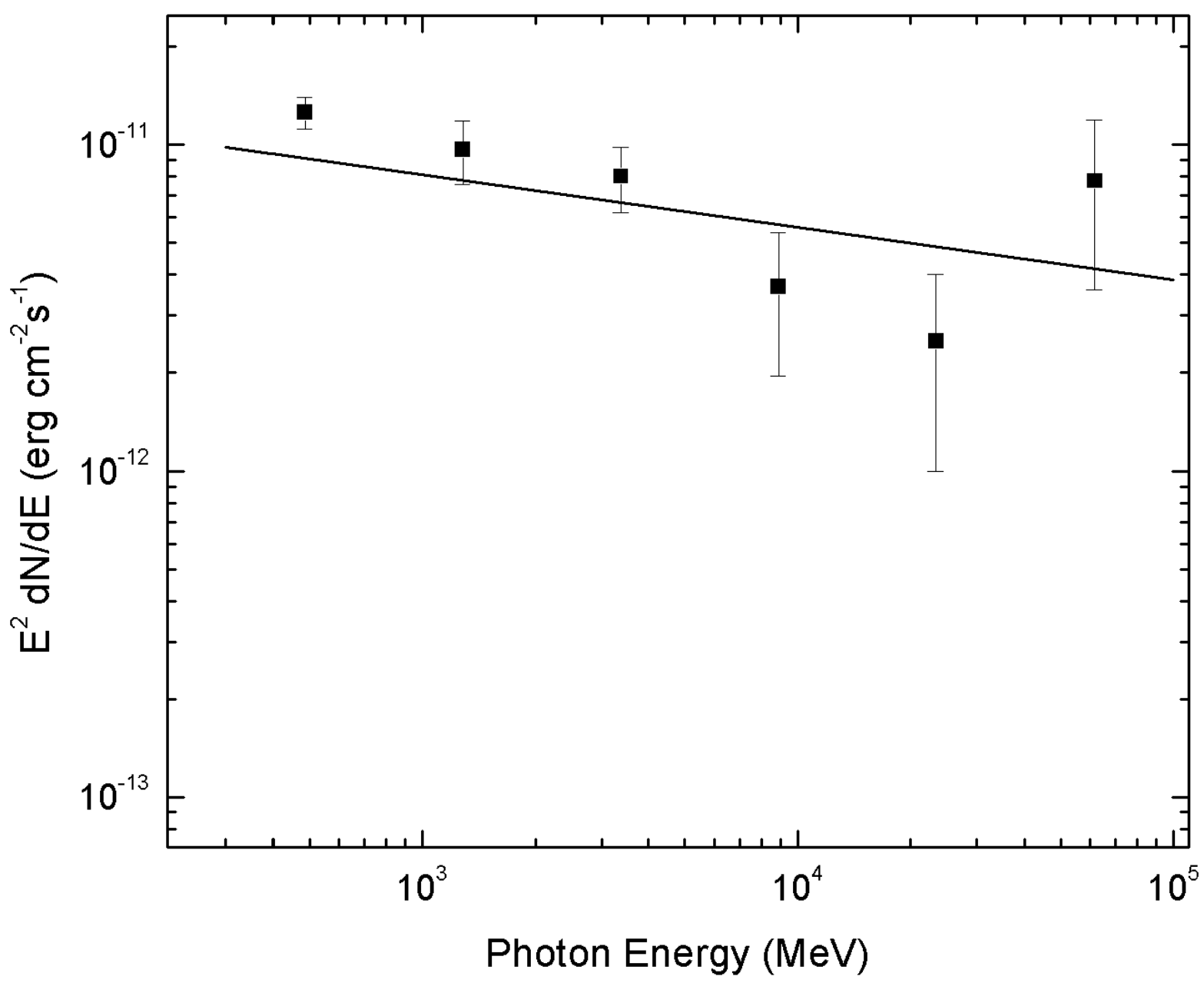

Fig. 5. - The spectrum $\left(E^{2} \times d N / d E\right)$ of the $\gamma$-ray emission found within the tidal radius of Liller 1. Photons between $300 \mathrm{MeV}$ and $100 \mathrm{GeV}$ are divided into 6 logarithmically-equally spaced energy bins and The derived flux for each bin is plotted at the corresponding mean energy. The solid line indicates the best-fit single power law model. The rightmost point has a TS value of 16 , corresponding to a significance level of four. 


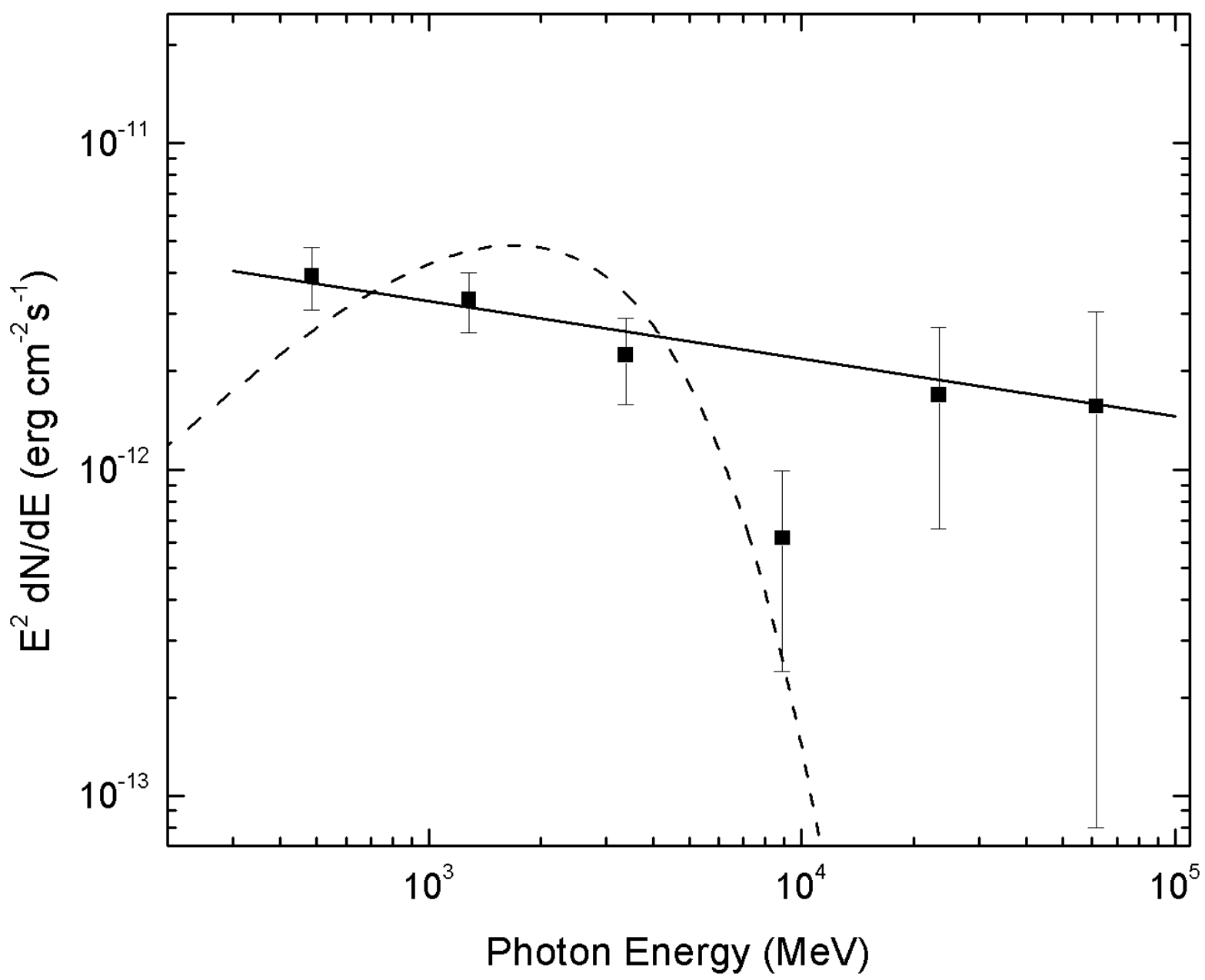

Fig. 6.- The spectrum $\left(E^{2} \times d N / d E\right)$ of the $\gamma$-ray emission found within the tidal radius of NGC 6624. Photons between $300 \mathrm{MeV}$ and $100 \mathrm{GeV}$ are divided into 6 logarithmicallyequally spaced energy bins and The derived flux for each bin is plotted at the corresponding mean energy. The solid line and the dashed line indicate the best-fit single power law model and the PLE model with $E_{\mathrm{c}}=1.5 \mathrm{GeV}$, respectively. Each of the rightmost two points has a TS value of $\sim 10$, corresponding to a significance level of about three each. 


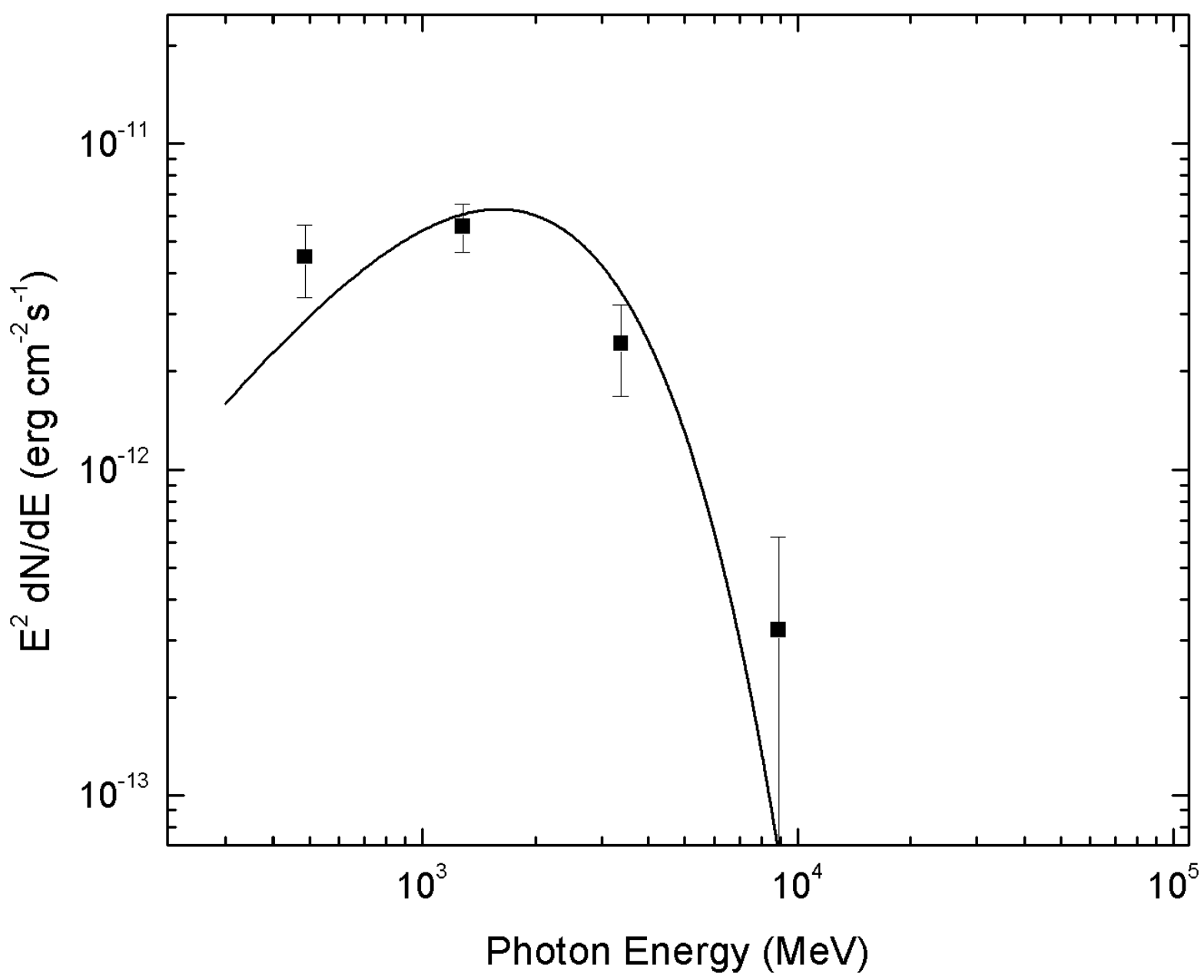

Fig. 7. - The spectrum $\left(E^{2} \times d N / d E\right)$ of the $\gamma$-ray emission outside the tidal radius of NGC 6441. The solid line indicates the best-fit PLE model. 